\title{
Study of the effect of different body positions on ocular vestibular-evoked myogenic potentials using air-conducted sound
}

\author{
Samir Asal ${ }^{\mathrm{a}}$, Ossama Sobhy ${ }^{\mathrm{a}}$, Nervana Salem ${ }^{\mathrm{b}}$
}

\author{
aDeparment of Audiovestibular Medicine, \\ Faculty of Medicine, University of Alexandria, \\ Department of Audiovestibular Medicine, \\ Alexandria Main University Hospital, \\ Alexandria, Egypt
}

Correspondence to Nervana Mohame Salem, MBBCH alex, M.Sc, Audiovestibular Medicine, Fifth Year Resident of Audiovestibular Medicine in Alexanderia Main University Hospital; e-mail: nervanasalem87@gmail.com

Received 8 February 2017

Accepted 18 April 2017

The Egyptian Journal of Otolaryngology 2017, 33:588-593

\begin{abstract}
Objective
The tested hypothesis states that by manipulating body position, a differentiation in the optimum body position for ocular vestibular-evoked myogenic potential (oVEMP) testing could be obtained.

Patients and methods

The present study was conducted on 33 ears ( 33 healthy adult volunteers) with no age or sex limit or any ontological complaint in the audiology unit of Alexandria Main University Hospital. Pure-tone audiometry, tympanometry, and oVEMP testing were performed. Ocular VEMP was performed in four different positions - sitting, supine, right decubitus, and left decubitus positions. Ocular VEMP waveforms were analyzed regarding morphology, latency, amplitude, and threshold.
\end{abstract}

Results

(a) OVEMP was present in $90 \%$ of the studied cases. (b) The sitting position produced the shortest latencies. (c) The independent position provided the largest amplitude. (d) The dependent position elicited the highest thresholds.

Conclusion

Although the best position for oVEMP test could not be determined by the present study, the trends found support that the sitting position may be preferred for future oVEMP testing based on the short latencies produced in this position. On the other hand, high thresholds were obtained in the dependent (left decubitus) position, which indicates that it is the least favorable position.

\section{Keywords:}

air-conducted sound, otoconial membrane, otolith organs, ocular vestibular-evoked myogenic potential, utricule

Egypt J Otolaryngol 33:588-593

(C) 2017 The Egyptian Journal of Otolaryngology

1012-5574

\section{Introduction}

Vestibular-evoked myogenic potential (VEMP) is a biphasic, short-latency muscle potential evoked by acoustic, bone, or galvanic stimulation. Thus, clinically, VEMP can assess dynamic otolithic function $[1,2]$. Otolith afferents from each utricular and saccular maculae can be stimulated by both airconduction stimulus (ACS) and bone-conduction stimulus $[3,4]$. The utriculo-ocular and sacculocollic neural connections are strong connections, whereas the motor projections from the saccule to the ocular system are weak [5]. Consequently, the saccule elicits cervical vestibular-evoked myogenic potential (cVEMP), whereas ocular vestibular-evoked myogenic potential (oVEMP) is elicited from the utricle [6]. Substantially, it is motor and not sensory specific [7]. Several studies have been carried out to analyze oVEMP findings as the oVEMP pathway has been confirmed.

The effect of body position on $\mathrm{CVEMP}$ has been studied previously, as cVEMP was comparatively discovered before oVEMP. The effect of saccular orientation on cVEMP in relation to gravity was studied in individuals with normal vestibular function. Manipulation of the upper body and head with visual fixation versus no visual fixation revealed that ipsilateral contraction of the sternocleidomastoid muscle (SCM) while sitting produced the greatest VEMP response with no effects from visual input [8]. Isaacson et al. [9] assessed the consequences of different methods of SCM activation on VEMP, and they concluded that increased tonic SCM electromyography activity resulted in increased amplitude in the supine with head turned position. Another study found no significant difference in P1 and $\mathrm{N} 1$ potentials in the upright and supine positions; therefore, effectively both positions can be used to record VEMP [10].

In this recently developed test, to elaborate standardized data, thorough investigations are being performed nowadays. Studies are being carried out on

This is an open access article distributed under the terms of the Creative Commons Attribution-NonCommercial-ShareAlike 3.0 License, which allows others to remix, tweak, and build upon the work noncommercially, as long as the author is credited and the new creations are licensed under the identical terms. 
threshold values and recording parameters. On the other hand, there is dearth in the field of the effect of body and head positions in different planes.

On the basis of the dual sensitivity of the otolith organs to both physiological (gravitational acceleration) and nonphysiological (acoustic AC and $\mathrm{BC}$ ) stimuli, the present study investigated oVEMP positioning techniques to boost future oVEMP procedures. The aim of this study was to disclose changes in oVEMP response when body position is variable. Given that the otolith maculae have completely different anatomical orientation, gravitational forces on the otoconial membranes of the utricle and saccule should give rise to differential gravity-specific resting potentials throughout specific body positions. The effect of different body positions on oVEMP using air conduction stimulation (ACS) was examined in this study.

\section{Patients and methods}

The present study was conducted on 33 ears (33, healthy, adult volunteers) in Alexandria Main University Hospital with no age or sex limit, no previous complaint of dizziness or imbalance, and no otological or neurological diseases. The methodology described by O'Neil [2] was used in this study with modifications according to device capabilities. Informed consent was obtained from all patients. In case of incompetent patients, consent was obtained from the guardians. This study was conducted after obtaining approval from the Ethics Committee of Alexandria Faculty of Medicine.

Ocular VEMP was recorded contralateral to acoustic stimulation of the left ear through standard electrode montage and recording parameters using disposable silver chloride electrodes with impedances maintained below $5 \mathrm{k} \Omega$.The active electrode was placed $1 \mathrm{~cm}$ infraorbitally, directly beneath it the reference electrode was placed, whereas the ground electrode was placed on the high forehead (Fpz).

GSI Audera auditory-evoked potential unit (GrasonStadler, Eden Prairie, Minnesota, USA) was used to acquire and average oVEMP activity using $500-\mathrm{Hz}$ tone burst delivered through headphones. An artifact rejection system was applied. The rate used was $5 \mathrm{~Hz}$ in 100 sweeps, with the following duration - two cycles plateau and one cycle rise/fall - and the intensity presented was 70-95 dBnHL. Rarefaction polarity and Blackman Ramping were used. The prestimulus/ post-stimulus time was $-10.5 \mathrm{~ms}$, and the filter used $5-750 \mathrm{~Hz}$ with amplification of 5000 times.
In the four positions, oVEMP testing was carried out with the stimulus being presented monaurally to the left ear as the patient contracted the inferior oblique (IO) muscle by elevating the eye $20^{\circ}$. In order to bring the utricle into the horizontal plane and to eliminate the saccule during testing, the patient's head was positioned so that the vestibular system was at a $30^{\circ}$ angle off the horizontal plane; therefore, in all positions, the chin was tilted $30^{\circ}$ toward the chest. For each patient, a randomized sequence of positions A, B, C, and D was used to avoid fatigue. Position A was sitting upright, position $\mathrm{B}$ was lying supine, position $\mathrm{C}$ was lying on the right side, and position $\mathrm{D}$ was lying on the left side.

The stimulus was presented at $95 \mathrm{dBnHL}$ for all participants and was duplicated to confirm both present and absent responses in all four positions. Between each pair of oVEMP trials, the patient was allowed to rest. In case no oVEMP was obtained at 95 $\mathrm{dBnHL}$, the recording session was ended, and the recordings were fed to the database as an absent response. If a response was obtained, the intensity was decreased by $5 \mathrm{dBnHL}$ until the oVEMP threshold was reached. To make sure the recordings were accurate, the threshold and the intensity level below the threshold were replicated. This procedure of finding thresholds was performed in all four positions.

Analysis parameters for all positions were Morphology of Waveform classified as intact and absent, N1 latency reflecting nerve conduction velocity, and amplitude and threshold reflecting excitability of the nerve. N1-P1 amplitude is presented in microvolts (peakto-peak amplitude), and threshold is the lowest intensity level at which a response to the stimulus appeared.

\section{Statistical analysis [11]}

Data were fed to a computer and analyzed using IBM SPSS software package (version 20.0; IBM Corp., Armonk, New York, USA) [12]. Qualitative data were described as numbers and percentages; minimum and maximum values; means and SDs; and medians. Significance was judged at the 5\% level. The used tests were analysis of variance with repeated measures, Friedman's test, and Wilcoxon's signed-rank test.

\section{Results}

The study was carried out on 33 volunteers. Twentyone of them were males and 12 were females. The age of patients ranged from 21 to 50 years with a mean age 
of $29.85 \pm 7.31$ years. Thirty cases had recordable oVEMP of 90.9\%, and three cases had no recordable oVEMP of $9.1 \%$.

\section{N1 latency}

Table 1 and Fig. 1 show comparison between different measures of N1 latency in all four positions. There was a statistically significant difference when comparing reflex N1 latency in the sitting and supine positions, where the sitting position produced shorter N1 latencies.

\section{Amplitude}

Table 2 and Fig. 2 show the comparison between different measures of amplitude in all four positions. There was no significant statistical

Figure 1

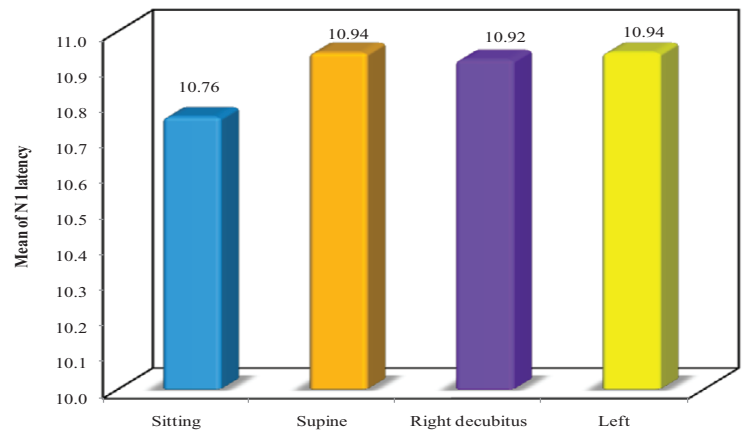

Comparison of $\mathrm{N} 1$ latency at different body positions. difference between the four positions, but trends were found to indicate that the independent position gives the largest amplitude. This means that stimulation of the upper ear produces the highest amplitude. Correlation was calculated for the median and not the mean because of the large $\mathrm{SD}$.

\section{Threshold}

Table 3 and Fig. 3 illustrate comparisons between different measures of threshold in all four positions. Significantly higher thresholds were recorded comparing the dependent position with the other three positions. This means that stimulation of the lower ear elicits the worst threshold response $(P=0.001)$.

\section{Figure 2}

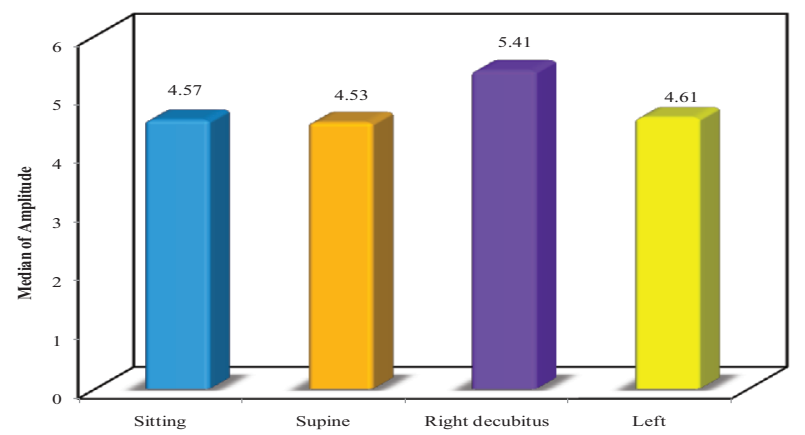

Comparison of amplitudes measured in the four studied positions.

Table 1 Results of N1 latency of ocular vestibular-evoked myogenic potential in different body positions (ms)

\begin{tabular}{lccccc}
\hline N1 latency & Sitting $(n=30)$ & Supine $(n=30)$ & Right decubitus $(n=30)$ & Left decubitus $(n=30)$ & $F$ \\
\hline Minimum-maximum & $9.84-12.84$ & $9.50-12.84$ & $9.50-13.17$ & $9.17-13.50$ & 0.985 \\
Mean \pm SD & $10.76 \pm 0.73$ & $10.94 \pm 0.77$ & $10.92 \pm 0.80$ & $10.94 \pm 0.86$ & \\
Median & 10.67 & 10.84 & 10.84 & 10.84 & \\
$P_{1}$ & & $0.046^{*}$ & 0.171 & 0.219 & \\
$P_{2}$ & & 0.878 & 0.976 & \\
$P_{3}$ & & & 0.907 & \\
\hline
\end{tabular}

$F, F$ test [analysis of variance (ANOVA)] with repeated measures. $P_{1}$, adjusted least significant difference (LSD) $P$-value for ANOVA with repeated measures for comparison between sitting and other postures. $P_{2}$, adjusted LSD $P$-value for ANOVA with repeated measures for comparison between supine and other postures. $P_{3}$, adjusted LSD $P$-value for ANOVA with repeated measures for comparison between right decubitus and left decubitus positions. "Statistically significant at $P \leq 0.05$.

Table 2 Results of N1-P1 amplitude in the four positions $(\mu \mathrm{V})$

\begin{tabular}{lccccc}
\hline Amplitude & Sitting $(n=30)$ & Supine $(n=30)$ & Right decubitus $(n=30)$ & Left decubitus $(n=30)$ & $\chi^{2}$ \\
\hline Minimum-maximum & $1.04-20.91$ & $1.27-25.96$ & $1.0-18.80$ & $0.51-14.71$ & 0.823 \\
Mean \pm SD & $5.21 \pm 4.13$ & $5.74 \pm 5.06$ & $5.53 \pm 3.98$ & 0.844 \\
Median & 4.57 & 4.53 & 5.41 & $4.98 \pm 3.44$ & \\
$P_{1}$ & & 0.154 & 0.495 & 0.61 & \\
$P_{2}$ & & & 0.658 & 0.696 & \\
$P_{3}$ & & & & 0.229 & \\
\hline
\end{tabular}

$\chi^{2}, \chi^{2}$ for Friedman's test. $P_{1}, P$-value for Wilcoxon's signed ranks test for comparing between sitting and other positions. $P_{2}$, $P$-value for Wilcoxon's signed ranks test for comparing between supine and other positions. $P_{3}, P$-value for Wilcoxon's signed ranks test for comparing between right decubitus and left decubitus positions. 
Table 3 Results of ocular vestibular-evoked myogenic potential threshold in the four positions (dBnHL)

\begin{tabular}{lccccc}
\hline Threshold & Sitting $(n=30)$ & Supine $(n=30)$ & Right decubitus $(n=30)$ & Left decubitus $(n=30)$ & $F$ \\
\hline Minimum-maximum & $80.0-95.0$ & $80.0-95.0$ & $80.0-95.0$ & $80.0-95.0$ & $5.661^{*}$ \\
Mean \pm SD & $86.67 \pm 4.01$ & $86.50 \pm 4.18$ & $87.17 \pm 3.64$ & $88.0 \pm \pm .07$ & \\
Median & 85.0 & 85.0 & 87.50 & 90.0 & \\
$P_{1}$ & & 0.662 & 0.184 & $0.003^{*}$ & \\
$P_{2}$ & & 0.103 & $0.005^{*}$ & \\
$P_{3}$ & & & $0.023^{*}$ & \\
\hline
\end{tabular}

$F, F$ test [analysis of variance (ANOVA)] with repeated measures. $P_{1}$, adjusted least significant difference (LSD) $P$-value for ANOVA with repeated measures for comparison between sitting and other postures. $P_{2}$, adjusted LSD $P$-value for ANOVA with repeated measures for comparison between supine and other postures. $P_{3}$, adjusted LSD $P$-value for ANOVA with repeated measures for comparison between right decubitus and left decubitus positions. "Statistically significant at $P \leq 0.05$.

Figure 3

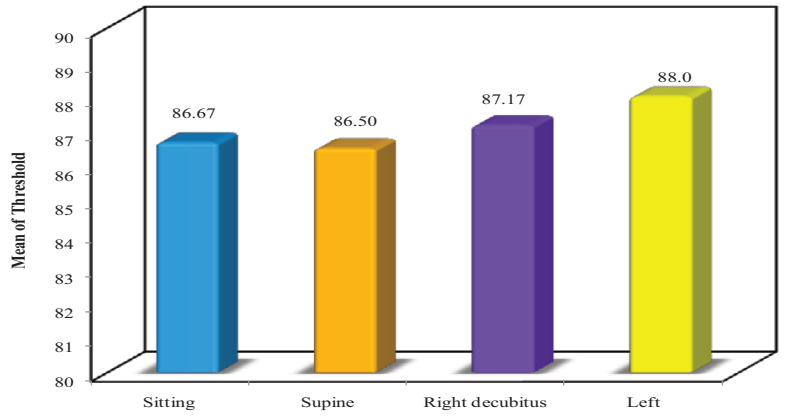

Comparison of thresholds measured in the four studied positions.

\section{Discussion \\ Waveforms}

No significant changes were detected regarding oVEMP waveform morphology in all four positions. All positions produced intact waveforms. The examination session was terminated for patients who had no response at $95 \mathrm{dBnHL}$ in all four positions, and data were entered into the database as having absent reflex. It was not expected that the presence or absence of waveforms will be affected by different body positions. On the other hand, minimal changes are expected to be found regarding other oVEMP response parameters when body position is a variable.

\section{Latency}

There was a statistically significant difference when comparing N1 latency in the sitting and supine positions, attributed to shorter N1 latency produced during the sitting position. Conjointly, an observed trend was found, indicating that the sitting position produced the shortest latencies compared with all other positions. The possible explanation is that, in the supine position, gravitational forces are aligned with the polarization vectors of saccular and not utricular hair cells, whereas in the sitting position gravitational forces will exert maximal resting potential on utricular hair cells. Consequently, this led to a significant difference between sitting and supine positions and conjointly explains the trend found where the sitting position produced the shortest N1 latency, which is the robust component of oVEMP.

\section{Amplitude and threshold}

In the dependent (left decubitus) position, higher thresholds were obtained, whereas in the independent (right decubitus) position a significant trend was found, indicating that higher amplitude can be recorded in this position. These two results can be attributed to a number of explanations.

First, ipsilateral head tilts (in relation to gravity) cause stimulation of the utricular receptors, which respond with an increased afferent firing rate [13]. On the contrary, contralateral head tilts have an opposite, but much weaker, effect on the activity of utricular afferents. On the basis of the hypothesis that the utriclar sensitivity to nonphysiological stimuli such as AC oVEMP might be hindered by increased resting discharge of irregular afferents from the dependent ear, resulting in masking the AC oVEMP reflex recorded from the contralateral eye, which is already occupied with the effect of gravitational shearing forces of head tilt, led to higher oVEMP thresholds from stimulation of the dependent ear. This subsequently explained the trend toward higher amplitude from stimulating the independent ear.

Second, the phenomenon of ocular counter-roll (OCR) should be taken into consideration. OCR represents a static vestibuloocular reflex effected by the IO muscle, in which ipsilateral head tilts tonically activate the utricule, resulting in excyclorotation in the contralateral (upper) eye. In addition, the major responsible muscle for the oVEMP is believed to be the IO muscle. In conclusion, the two vestibuloocular reflexes - the VEMP and the OCR - evolve within the vestibulum and share the IO muscle as an effector. Therefore, the increased baseline muscle activity 
caused by the OCR owing to head tilt will result in occupation of the IO, thus decreasing the sensitivity of the dependent utricle to ACS. This is in contrast with Govender et al. [14] and Rosengren et al. [15] who proposed that a higher baseline IO muscle activity produces larger oVEMP responses, which has been observed to be the case with cVEMP [16].

Third, Shojaku et al. [17] further suggested that the weight of the otoconial membrane might affect the VEMP amplitudes. His suggestion was based on the concept that during head tilt $\pm 90^{\circ}$, the mechanical loading on utricular hair cells is maximal because the utricular macula is brought into the plane of the gravitational axis. Therefore, in this position, the capacity for further hair cell displacement by stapes motion will be limited [17].

Fourth, a more reasonable explanation was projected in a recent study on the effect of head and body tilt in roll plane [18], which may also explain our results that oVEMP responses to AC sound are selectively affected by mechanical constrains imposed by change in head position. Increased intracranial pressure subsequently increases intracochlear pressure that might impede sound transmission through the middle ear. These effects are likely to be more pronounced for the dependent ear, where great pressure is exerted on the cochlear windows and stapes motion is directed against gravity.

Interestingly, Iwasaki et al. [19] studied different effects of head tilt on oVEMP in response to boneconducted vibration and air-conducted sound. They have shown that head tilt in the roll plane increased the asymmetry ratios of oVEMPs to bone conduction vibration $(\mathrm{BCV})$, and they attributed their results to the concept that activation of the utricle changes according to the changes in gravitational shear forces, thus affecting oVEMP response [19].

On the other hand, head tilt failed to have a significant effect on either the asymmetry ratios or latencies of oVEMPs to ACS. The authors failed to find the same effect for ACS oVEMP as in our study because their experiment was different. Iwasaki et al. [19] instructed patients to tilt their head $90^{\circ}$ by tilting the neck as well as the body. They usually tilted the neck by $\sim 45^{\circ}$ and the body by $\sim 45^{\circ}$. With this maneuver, they modified not only the position of the otolithic maculae in relation to gravitational force, but also conjointly the relative position of the head versus the trunk, thereby presumably changing the hydrostatic pressure of cranial cerebrospinal fluid and the inner ear. This may have confounded the modulation effect in their experiment. ACS is a completely different stimulus from $\mathrm{BCV}$, in which ACS oVEMPs are likely to be more prone to hydrostatic changes of the cerebrospinal fluid/the inner ear. We avoided this possible confounding factor by comparing the positions sitting versus supine versus right side down versus left side down, where the head was always on the same level as the trunk.

Previous studies have used AC stimuli to analyze the consequences of head position on the cVEMP. Ito et al. [20] studied patients in the upright, supine, prone, and left and right lateral positions, and they found no significant amendment in corrected cVEMP amplitudes. A significant difference in baseline sacculus afferent activity was not expected when comparing the dependent ear and the independent ear positions, because the saccular macula lies flat and perpendicular to the gravitational force vector in both positions [20].

All the above-mentioned studies [18-21] solely examined the changes in amplitude and failed to examine threshold changes; however, their explanations match the results of this study relating to the amplitude and threshold results. One study examined the effect of body position on oVEMP threshold. Although no applied statistical significance was found, trends were observed in their data. The generalized estimating equation (GEE) statistical analysis resulted in trends indicating that having participants sit upright throughout oVEMP testing is optimum for achieving the best threshold possible. When evaluating which position induced a participant's best threshold, sitting upright was the best position for $73 \%$ of the observations. This is compared to lying supine, lying on the right side, and lying on the left side, which all elicited the best threshold response for $\sim 50 \%$ of the observations [2]. Although this study recommended the sitting position for future oVEMP testing, the absence of oVEMP in certain positions could not be clearly explained. In addition, a different age group was included in this study, which might justify their results.

\section{Conclusions and recommendations}

oVEMP was present in $90 \%$ of the studied cases. The sitting position produces the shortest latencies, the independent position provides the largest amplitude, and the dependent position evoked the highest thresholds.

This study experienced limitations with sample size. A total of 33 ears were evaluated, but little variability was obtained in the results. This could be attributed to the 
small changes in oVEMP due to the effect of position changing or the small sample size. Therefore, additional studies on positional testing are warranted to detect the effect of position changing during oVEMP testing.

The statistically significant results and trends found in our study may support the idea that the upright sitting position is preferred for future oVEMP testing and that the left decubitus (dependent) position is the least favorable position.

\section{Financial support and sponsorship}

Nil.

\section{Conflicts of interest}

There are no conflicts of interest.

\section{References}

1 Akin FW, Murnane OD. Vestibular evoked myogenic potentials: preliminary report. J Am Acad Audiol 2001; 12:445-452; quiz 91.

2 O'Neil A. Ocular vestibular evoked myogenic potentials (oVEMP) using air conducted sound: effect of body position on threshold. Alexandria, Egypt: Washington University School of Medicine; 2011.

3 Curthoys IS, Vulovic V. Vestibular primary afferent responses to sound and vibration in the guinea pig. Exp Brain Res 2011; 210:347-352.

4 Curthoys IS, Vulovic V, Manzari L. Ocular vestibular-evoked myogenic potential (oVEMP) to test utricular function: neural and oculomotor evidence. Acta Otorhinolaryngol Ital 2012; 32:41-45.

5 Uchino Y, Kushiro K. Differences between otolith- and semicircular canalactivated neural circuitry in the vestibular system. Neurosci Res 2011; 71:315-327.

6 Kantner C, Gurkov R. Characteristics and clinical applications of ocular vestibular evoked myogenic potentials. Hear Res 2012; 294:55-63.

7 Curthoys IS, Manzari L, Smulders YE, Burgess AM. A review of the scientific basis and practical application of a new test of utricular function - ocular vestibular-evoked myogenic potentials to boneconducted vibration. Acta Otorhinolaryngol Ital 2009; 29:179-186.

8 Sadler JH. The effects of body position and visual fixation on the vestibular evoked myogenic potential [dissertation/thesis]. Virginia: James Madison University; 2007.

9 Isaacson B, Murphy E, Cohen H. Does the method of sternocleidomastoid muscle activation affect the vestibular evoked myogenic potential response? J Vestib Res 2006; 16:187-191.

10 Pai RA, Bhat JS. Effect of body posture on vestibular evoked myogenic potentials. IJSR 2013; 2:396-398.

11 Kotz S, Balakrishnan N, Read CB, Vidakovic B. Encyclopedia of statistical sciences. 2nd ed. Hoboken, NJ: Wiley-Interscience; 2006.

12 Kirkpatrick LA, Feeney BC. A simple guide to IBM SPSS statistics for version 20.0. Student ed. Belmont, CA: Wadsworth, Cengage Learning; 2013

13 Fernandez C, Goldberg JM, Abend WK. Response to static tilts of peripheral neurons innervating otolith organs of the squirrel monkey. $J$ Neurophysiol 1972; 35:978-987.

14 Govender S, Rosengren SM, Colebatch JG. The effect of gaze direction on the ocular vestibular evoked myogenic potential produced by air-conducted sound. Clin Neurophysiol 2009; 120:1386-1391.

15 Rosengren SM, Colebatch JG, Straumann D, Weber KP. Why do oVEMPs become larger when you look up? Explaining the effect of gaze elevation on the ocular vestibular evoked myogenic potential. Clin Neurophysiol 2013; 124:785-791.

16 Colebatch JG, Halmagyi GM, Skuse NF. Myogenic potentials generated by a click-evoked vestibulocollic reflex. J Neurol Neurosurg Psychiatry 1994; 57:190-197.

17 Shojaku H, Watanabe Y, Tsubota M, Katayama N. Evaluation of the vestibular evoked myogenic potential during parabolic flight in humans. Exp Brain Res 2008; 187:477-481.

18 Taylor RL, Xing M, Black DA, Halmagyi GM, Welgampola MS. Ocula vestibular evoked myogenic potentials: the effect of head and body tilt in the roll plane. Clin Neurophysiol 2014; 125: $627-634$

19 Iwasaki S, Chihara Y, Egami N, Fujimoto C, Murofushi T, Yamasoba T. Different effects of head tilt on ocular vestibular-evoked myogenic potentials in response to bone-conducted vibration and air-conducted sound. Exp Brain Res 2012; 223:389-396.

20 Ito K, Karino S, Murofushi T. Effect of head position on vestibular evoked myogenic potentials with toneburst stimuli. Acta Otolaryngol 2007; 127:57-61.

21 Gurkov R, Kantner C. Modulation of oVEMP amplitudes by lateral head tilts. Clin Neurophysiol 2013; 124:1911-1912. 